\title{
Study of Substation Equipment State Monitoring System and Integrating Platform
}

\author{
Xu Xiaohui ${ }^{1}$, Ruixiang Fan², Benren Pan², Jianbo Xin², Danna Wang ${ }^{3}$ \\ 1.China Electric Power Research Institue, Nanjing 210003,China \\ 2.Jiangxi Electric Power Research Institute, NanChang 330096,China \\ 3. Hohai University, Nanjing 210098, China
}

Keywords: state monitoring system; information model; IEC 61850; IEC 61970; substation

\begin{abstract}
An information architecture of state monitoring substation equipment system is proposed based on IEC 61850 and IEC 61970. Asubstation equipment state monitoring and integrating platform (CMIP) supporting the unified information model and unified communication interface is designed and implemented. In this platform, comprehensive condition information of both the primary equipment and secondary equipment can be conveniently gathered and shared with other application systems.
\end{abstract}

\section{Introduction}

These years the technology of substation equipment state monitoring and condition repairing is gradually carried out and achieved great results [1].However,prominent problems are still exists:Firstly, state monitoring equipment inside substation can't achieve standardization. At present state monitoring system is accomplished by different company. They hold different comprehension so the interface and function are different,it's difficult to mutual operation and mutual replace [1-3]. Secondly,state monitoring system share. Today it's difficult to use different message for evaluating equipment's condition and life prediction because data message model and single communication interface between electrical company's control center and each substation are highly [1-3]. Due to the contradiction between field of these devices harsh operation environment and the level of the manufacturing process, the false report occurs. So establishment of a set of standard substation equipment monitoring system for the comprehensive assessment of the health status of substation equipment has important significance [4].

Because of the different between IEC61850 and IEC61970 standard, the unified modeling and seamless communication of power system became IEC TC's target [5]. It's also the direction of domestic and foreign experts to explore and get the corresponding results.But,as for equipment state monitoring system, the two standards have shortcomings. At present, there is no standard application in the state monitoring system. Only article puts forward the monitor information considers SCADA system and Energy manager system.And it is pointed out that significant to identify the network topology and improve the robustness of the power system.

This paper analyzes the construction of the equipment information model,such as assets management and evaluation, and use the crucial technology in the practical application.

\section{Structure of state monitor system}

The equipment condition information collection of the substation equipment is the basis of equipment state evaluation, risk assessment,maintenance strategy formulation and maintenance strategy formulation [6]. Equipment status information includes online information and offline information. 


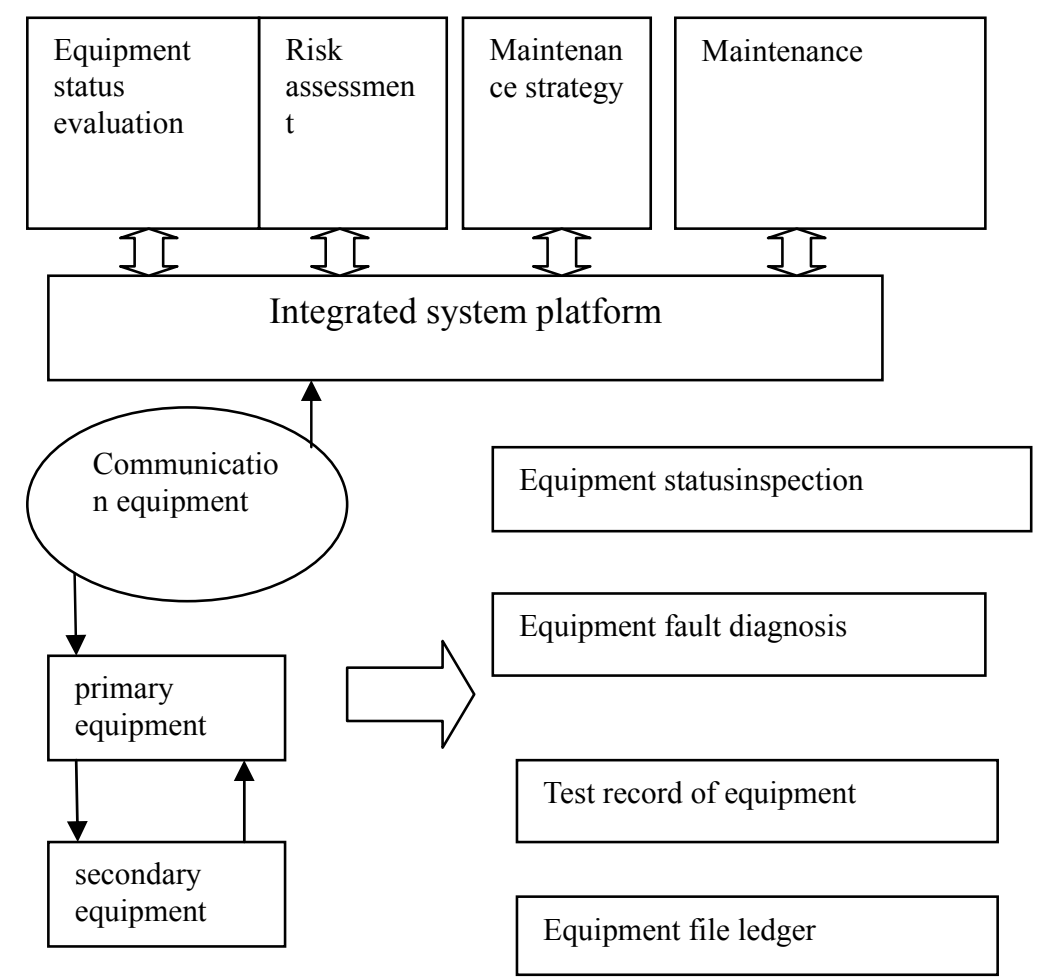

Fig. 1 Architecture of condition monitoring information integrated system of substation equipment

The online information mainly points operating equipment's online condition information, collecting equipment's online condition based on standard IEC61850, offline information contains equipment archives, install record, maintenance and so on[7-9]. Online information and offline information coordinate becomes the crucial technology of the development for substation equipment condition monitoring.

\section{Build the model of substation equipment state monitoring system}

\subsection{State model of typical equipment in IEC 61850}

IEC 61850 only defines four logical nodes that only used for monitoring high voltage equipment: STML,SIMG,SARC,SPDC. With the development of condition monitoring and sensor technology, the data of these logical nodes can't satisfy needs. So we need to establish model for once equipment and twice equipment, in order to expansion data under the standard IEC61850.

SCBR,SENV,SDGA,INTP,ICTP,ISTP belongs to undefined LN in IEC61850. SCBR achieve monitoring electrical and mechanic state characteristic of the circuit breaker. SCNV achieve monitoring temperature, humidity and air pressure [10-12]. SDGA achieve according to the oil-immersed transformer state of dissolved gas analysis in oil is the most common used device to create logical node. Achieve the gas in oil curve records and the function of the volume. In addition, although logical node not specifically used for monitoring state, it contains mass message that close to once and twice device status such as CCGR achieves monitoring and controlling oil temperature of transformer.

\subsection{IEC 61850 and 61970 information model adaptation}

In addition to the logic nodes that are specifically used for condition monitoring, 61850 IEC is actually defined as information that can be used to represent the state of a general device. Figure 2 is an example of a circuit breaker XCBR model logical node, which illustrates the adaptation of the general IEC 61850 logical node model to the public information model.

In order to evaluate the equipment status, the status model of system resources is needed. The model of CIM12R02 is defined in Asset Basics in IEC61968 [12-14]. It is defined as the current state and time of 2 attributes, and the mapping relationship is established with the IEC 61850 information, as shown in Figure 5. The evaluation theory (reason) and the (assessor) in the IEC 61850 model do not have the corresponding information, which need to automatically generate or 
evaluate the interface input based on the information source in the model mapping. In order to make computer intelligent system and evaluate experts to judge equipment state, we classify online equipment into 5 categories: excellent, good, general, notice, danger.

\begin{tabular}{|l||l||l|}
\hline Status type & PSR status & Health \\
Good:integer & Current status & D: string \\
Well: integer & Date time: date & T:timestamp \\
Normal: & time & stval: integer \\
integer & Status type: type & du: string \\
Warn: integer & Remarks: string & q:quality \\
Danger: integer & reason: integer & 61850 \\
& quality: string & \\
& & \\
& & \\
& & \\
& & \\
\end{tabular}

Fig. 2 Extension of CIM on condition monitoring and information mapping with IEC 61850 health model

\section{System interface design and implementation}

\section{1 system information interface design}

Transformer substation equipment online monitoring system is composed of platform and central level monitoring system. The system interface is shown in Figure 6.The crucial technology to realize the integrated platform for monitoring in substation:

1) IEC 61850 standard and not IEC 61850 standard state monitoring device access, and the IEC 61850 standard unified modeling.

2) The data is transmitted to the central level equipment monitoring platform based on the IEC 61850 or the transparent forwarding mode.

3) To provide access interface services and Web interface with IEC 61850, and the background of substation local diagnosis.

The central level monitoring platform is implemented in 3 ways: the MMS based model information transmission; the MMS based file transfer; and the TCP/IP based transparent communication, which is used to adapt to the current situation of the IEC 61850. From the substation based on the SCL configuration file, Com trade curve data file, these files through the specific file parsing plug-in adapter to extend CIM.

\subsection{System application implementation}

The substation equipment condition monitoring system based on IEC 61850 and IEC 61970 standards are used in a $500 \mathrm{kV}$ substation and a $110 \mathrm{kV}$ substation in Jiangxi. Take a $110 \mathrm{kV}$ substation in Jiangxi as an example, the application scheme is shown in Figure 7. The integrated platform for monitoring the status of the equipment in the substation realizes the information integration of the different equipment. The circuit breaker monitoring device is connected with the IEC 61850, the transformer oil gas and the micro water monitoring unit, the weather station, and the voltage monitoring unit are connected with the communication protocol based on the RS-485 communication protocol. The system is based on IEC 61850. The central level of equipment status monitoring integrated platform for enterprise level monitoring information integration, the communication server in the MMS client, the realization and the substation internal state monitoring platform based on IEC 61850 communications, and to realize the transmission and analysis of the substation Com trade file and IED configuration file. The general data access (GDA) interface based on CIS (DMIS) is implemented, which includes OMS interface, which is used to monitor the status of a circuit breaker. Substation monitoring platform and collaboration to achieve transparent forwarding agent mechanism can greatly facilitate the different manufacturers of spacer device of remote diagnosis and maintenance.

\section{Conclusions}

This paper mainly analyzes the coordination of IEC 61850 and IEC 61970 series standards in the 
condition monitoring system, and realizes the key technology in the practical application. With the development of smart grid and digital substation and the improvement of the level of intelligent substation equipment, the self-description and self-diagnostic ability of the equipment are increasing, and the status information of substation equipment is more valuable. In this paper, the online monitoring system of substation equipment will be more able to reflect this advantage, the unified standard of all kinds of equipment in the substation "no blind spot" comprehensive monitoring can be achieved.

\section{Acknowledgements}

The authors would like to give their gratitude to the support of project "Research and demonstration of comprehensive integration technology for smart grid based on the feature of low carbon grid", which is a 2012 technology project from China state grid, and also a major project (2013BAA01B00) from Eleven the Five national science and technology support program.

\section{Reference}

[1].MCDONALD J D. Substation automation: IED integration and availability of information. IEEE PowerandEnergyMagazine,2003, 1(2) : 22-31.

[2].SUN Caixin. Present situation and development of condition online monitoring and diagnosis technology for power transmission and transformation equipment. Electric Power, 2005, 38(2):1 7.

[3].ZOU Jianming. Application of online monitoring technology on power grid. High Voltage Engineering, 2007, 33( 8) : 203206.

[4].JU Ping, ZUO Yingfei, WEN Fushuan, et al. Health diagnosisof power systems. Electric Power Automation Equipment,2004, 24(6) : 2225.

[5].LIU Guoding, WANG Like. 2005 IEC T C 57 annual meetingand recent work of SAC/ T C 82. Automation of Electric Power System s, 2006, 30( 5) : 14.

[6].TAN Wenshu, LIU Guoding, LIU Peiju an, etal. Introduction of 2003 IEC T C 57 annual meeting. Automation of ElectricPower Systems , 2004, 28( 4) : 13.

[7].WANG Dewen, ZHU Yongli, DI Jian, et al. A method forelectric power equipment remote control based on IEC 61850.Automation ofElectric PowerSystem s, 2009, 33( 5) : 50-54.

[8].ZHANG Jianmin, ZH U Bingquan, JIANG Yueliang, et al.Key issues in the design of master station of relay protectionand fault information system. Automation of Electric PowerSystem s, 2003, 27( 14) : 72-74.

[9].KOS TIC T , PREISS O, FREI C. Towards the formalintegration of two upcoming standards : IEC 61970 an d IE C61850/ / Proceedings of the Large Engineering SystemsConference on Power Engineering, M ay 7 9, 2003, Montreal,Canada: 24- 29.

[10].KE ZU NOVI M , DJOKI T , KOST I T. Automated monitoring and control using new data integration paradigm / / Proceedings of the 38th Annual Hawaii International Conference on System Sciences( H IC SS 05 ), January 3 6, 2005 , Big Island, HI, USA.

[11].PREISS O, KOS TIC T. Unified in formation models in support of location transparency for future utility applications / /Proceedings of the 39th Hawaii International Conference onSystem Sciences ( HICS S 06), January 47, 2006, Kauai, H I,USA.

[12].LIU Ming, HE Guangyu. Coordination between IEC 61850and IEC 61970 for the protection model. Automation ofElectric Power Systems , 2006, 30( 22) : 711.

[13].HU Liang, WANG Qian. Research on seamlesscommunicationsystem based on IEC 61850 and 61970.Telecommunications for Electric Power System, 2007,28(182) : 15- 20.

[14].ZH U Chengzhi, GU O C huangxin, SU N M in, et al. Powertrans former condition assessment based on improved evidential reasoning. High Voltage Engineering, 2008, 34 (11) : 2332 -2337. 